\title{
Risk of pneumonia with budesonide-containing treatments in COPD: an individual patient-level pooled analysis of interventional studies
}

This article was published in the following Dove Press journal:

International Journal of COPD

5 April 2017

Number of times this article has been viewed

\author{
Sally Hollis' \\ Carin Jorup ${ }^{2}$ \\ Dan Lythgoe ${ }^{3}$ \\ Gunnar Martensson ${ }^{2}$ \\ Pontus Regnell ${ }^{2}$ \\ Göran Eckerwall ${ }^{2}$
}

'AstraZeneca R\&D, Alderley Park, Macclesfield, UK; ${ }^{2}$ AstraZeneca

R\&D, Gothenburg, Sweden; ${ }^{3}$ Phastar,

Chiswick, London, UK
Correspondence: Sally Hollis

Centre for Biostatistics, Institute of Population Health, University of Manchester, Manchester Academic Health Science Center, Oxford Road, Manchester MI 3 9PL, UK

Tel +44 I6I 275766 |

Fax +44 I6I 2755205

Email sally.hollis@manchester.ac.uk
Background: Concerns have been raised that treatment of COPD with inhaled corticosteroids may increase pneumonia risk. Responding to a request from the European Medicines Agency Pharmacovigilance Risk Assessment Committee, a pooled analysis of interventional studies compared pneumonia risk with inhaled budesonide-containing versus non-budesonide-containing treatments and the impact of other clinically relevant factors.

Methods: AstraZeneca-sponsored, parallel-group, double-blind, randomized controlled trials meeting the following criteria were included: $>8$ weeks' duration; $\geq 60$ patients with COPD; inhaled budesonide treatment arm (budesonide/formoterol or budesonide); and non-budesonidecontaining comparator arm (formoterol or placebo). Primary and secondary outcomes were time to first pneumonia treatment-emergent serious adverse event (TESAE) and treatmentemergent adverse event (TEAEs), respectively, analyzed using Cox regression models stratified by study.

Results: Eleven studies were identified; 10,570 out of 10,574 randomized patients receiving $\geq 1$ dose of study treatment were included for safety analysis (budesonide-containing, $\mathrm{n}=5,750$; non-budesonide-containing, $\mathrm{n}=4,820$ ). Maximum exposure to treatment was 48 months. The overall pooled hazard ratio (HR), comparing budesonide versus non-budesonide-containing treatments, was 1.15 for pneumonia TESAEs ( $95 \%$ confidence interval [CI]: $0.83,1.57$ ) and 1.13 for pneumonia TEAEs (95\% CI: 0.94, 1.36). The annual incidence of pneumonia TESAEs was $1.9 \%$ and $1.5 \%$ for budesonide-containing and non-budesonide-containing treatments, respectively. Comparing budesonide/formoterol with non-budesonide-containing treatment, the HRs for pneumonia TESAEs and TEAEs were 1.00 (95\% CI: 0.69, 1.44) and 1.21 (95\% CI: 0.93, 1.57), respectively. For budesonide versus placebo, HRs were 1.57 for pneumonia TESAEs (95\% CI: 0.90, 2.74) and 1.07 for pneumonia TEAEs (95\% CI: 0.83, 1.38).

Conclusion: This pooled analysis found no statistically significant increase in overall risk for pneumonia TESAEs or TEAEs with budesonide-containing versus non-budesonide-containing treatments. However, a small increase in risk with budesonide-containing treatment cannot be ruled out; there is considerable heterogeneity in study designs and patient characteristics, particularly in the early budesonide studies, and each study contributes $<40$ pneumonia TESAEs.

Keywords: budesonide, COPD, inhaled corticosteroid, pneumonia

\section{Introduction}

Combination therapy of an inhaled corticosteroid (ICS) with a long-acting $\beta_{2}$-agonist (LABA) is widely used in the treatment of $\mathrm{COPD}^{1}$ to reduce the risk of exacerbation and improve lung function and quality of life..$^{1-3}$ Indeed, for those patients at high risk 
of an exacerbation ( $\geq 2$ exacerbations per year or $\geq 1$ hospitalization for exacerbation per year), combined ICS/LABA therapy is a recommended treatment option (Global Initiative for Chronic Obstructive Lung Disease; GOLD). ${ }^{1}$

However, in long-term COPD studies, patients treated with an ICS had higher rates of pneumonia than those receiving placebo, ${ }^{4,5}$ raising concerns that ICS treatment may increase pneumonia risk. This risk may vary between different ICSs, with some evidence of a weaker observed association with pneumonia for budesonide than for fluticasone. One epidemiological study directly compared budesonide with non-ICS treatments in a Canadian, newuser COPD population. ${ }^{6}$ In this nested case-control analysis of 163,514 COPD patients, ICS use (either alone or in a combination inhaler) was associated with a $69 \%$ increase in the risk of serious pneumonia (either requiring hospitalization or fatal), and this was higher with fluticasone (122\% increase at $1,000 \mu \mathrm{g} / \mathrm{d})$ than with budesonide (17\% increase). ${ }^{6}$ This finding was supported by a later study from the same group indicating a decrease in risk after discontinuation of ICS, including after discontinuation of budesonide. ${ }^{7}$ Recently, a comprehensive study-level meta-analysis from Kew and Seniukovich ${ }^{8}$ estimated the odds ratio for nonfatal pneumonia serious adverse events (SAEs) to be 1.62 for budesonidecontaining compared to non-budesonide-containing treatment (95\% confidence interval $[\mathrm{CI}]$ : 1.00-2.62).

In response to a request from the European Medicines Agency Pharmacovigilance Risk Assessment Committee, ${ }^{9}$ a further individual patient-level pooled analysis was conducted using available interventional studies in patients with COPD to compare the risk of pneumonia between budesonide-containing and non-budesonide-containing treatments. The analysis also aimed to address the extent to which any effect of budesonide on pneumonia risk is modified by other clinically relevant factors.

\section{Methods}

\section{Study selection}

This individual, patient-level, pooled analysis included all AstraZeneca-sponsored parallel-group, double-blind, randomized controlled trials of budesonide-containing treatments conducted before May 29, 2015 that met the following criteria: 1) $>8$ weeks' duration, 2) $\geq 60$ patients with COPD, 3 ) an inhaled budesonide treatment arm (ie, budesonide [Pulmicort, AstraZeneca] or budesonide/formoterol [Symbicort, AstraZeneca]), and 4) a non-budesonide-containing comparator treatment arm (ie, formoterol or placebo). Because this paper reports a pooled analysis of previous clinical trials ethical approval was obtained in the context of each trial, but not for this study.

\section{End points and assessments}

The primary outcome variable was time to first pneumonia treatment-emergent serious adverse event (TESAE) during the follow-up period; a 'serious' pneumonia event was one meeting the regulatory definition of an SAE, ${ }^{10}$ including hospital admission. Time to first TESAE was defined as time in days from date of first dose of randomized treatment to date of first pneumonia TESAE, or censored on the date of last dose of randomized treatment +14 days for patients who did not experience a pneumonia TESAE. Exposure was defined as time from date of first dose of randomized treatment to date of last dose of randomized treatment. Secondary outcome variables evaluated the time to first pneumonia treatment-emergent adverse event (TEAE), defined similarly to the primary outcome variable, and the total number of pneumonia TESAEs and TEAEs occurring during the follow-up period (defined as for the primary outcome variable).

In addition to the treatment comparisons for the effect of budesonide, the study also assessed whether other clinically relevant factors (such as lung function, smoking status, age, body mass index [BMI], and gender) modified the effect of budesonide-containing treatments on the risk of pneumonia TESAEs and TEAEs in COPD patients. The direct effect of these other clinically relevant factors on the risk of pneumonia TESAEs and TEAEs in COPD patients was also assessed (not reported here).

Pneumonia events were identified based on the AEs and SAEs reported by the investigator, using the MedDRA (version 18.0, IFPMA, Geneva, Switzerland) preferred terms considered relevant for community-acquired pneumonias associated with ICS use (Table S1). Pneumonia events were reported, and data were handled in accordance with the clinical practice and clinical trial methodology current at the time of each study. Viral or fungal pneumonias were excluded, as they are not considered to be typical communityacquired pneumonias relevant for this assessment.

\section{Statistical analysis}

All analyses were based on the safety analysis set, defined as all patients who were randomized and received at least one dose of study treatment. All analyses were conducted according to actual, rather than randomized, treatment. Data from all identified studies were pooled into a single database. For time to first TESAE and TEAE, Cox regression models 
stratified by study were used, allowing for a varying baseline hazard across studies.

The primary comparison for both TESAEs and TEAEs was budesonide-containing versus non-budesonide-containing treatments. For the relevant subset of studies in each case, the following secondary treatment comparisons were also made:

- A: budesonide + formoterol versus formoterol

- B: budesonide + formoterol versus placebo

- A + B: budesonide + formoterol versus non-budesonidecontaining

- $\mathrm{C}$ : budesonide versus placebo.

Relevant treatment arms were combined for the purposes of the primary and secondary treatment comparisons, with separate models used to obtain the secondary comparisons.

The assumption of proportional hazards was assessed for each of the primary and secondary comparisons using timedependent covariates, by adding a treatment by $\log _{\mathrm{e}}$ (time) interaction term to each model. For the primary comparison, the effect of treatment on the risk of a TESAE over time was also assessed using a piecewise Cox model. For this analysis, the time period was partitioned into $0-<3,3-<6$, 6-12, and $\geq 12$ months.

Comparisons of treatment arms within each study are also reported and were obtained using study-specific Cox regression models. As a sensitivity analysis, primary, secondary, and study-specific comparisons were repeated using models adjusted for all of the risk factors considered.

Heterogeneity in the forest plots of the treatment effect was assessed qualitatively by grouping studies by treatment comparison, with separate grouping for budesonidecontaining treatment dose, study treatment duration, and budesonide-containing treatment device. In a post hoc analysis, quantitative evidence of statistical heterogeneity was assessed by adding a treatment-by-stratum interaction term to each model and using a likelihood ratio test to compare the fit of respective models. ${ }^{11}$ Furthermore, the primary and secondary comparisons were reanalyzed using two-stage fixed effect meta-analysis in order to obtain $I^{2}$ statistics and corresponding 95\% CIs. ${ }^{12}$

Interaction effects were used to explore the extent to which the effect of the primary comparison of budesonide-containing versus non-budesonide-containing treatment was consistent across subgroups of the pooled dataset based on lung function (postbronchodilator [BD] forced expiratory volume in one second $\left[\mathrm{FEV}_{1}\right] \%$ predicted normal $[\mathrm{PN}]$ ), smoking status, age, BMI, and gender. For each factor, a Cox model was fit to the pooled data with the addition of the treatment and study as main effects and a treatment-by-factor interaction term. To assess the effect of each of the risk factors on TESAE and
TEAE risk, each factor was included, in turn, in a Cox model adjusted for treatment and study as main effects. As a sensitivity analysis, a single model including all factors simultaneously was used to obtain adjusted factor effects, again with additional adjustment for study and treatment as main effects.

All statistical tests were two-sided, and parameter estimates were presented with accompanying 95\% Wald-type CIs and illustrated using forest plots. $P$-values $<0.05$ were considered to be statistically significant, with the exception of interaction effects and heterogeneity tests, for which $P<0.10$ was considered to be statistically significant. No corrections were made for multiple comparisons.

\section{Post hoc sensitivity analysis}

Post hoc sensitivity analyses of primary, secondary, and studyspecific treatment comparisons were performed to first exclude patients with post-BD $\mathrm{FEV}_{1} \geq 80 \% \mathrm{PN}$ and then those with $\geq 70 \%$ to exclude patients who were unlikely to be treated with ICS according to current treatment guidelines. ${ }^{1}$ In a separate post hoc sensitivity analysis, patients treated in centers in Denmark were excluded due to increased reporting of pneumonia as a nonserious AE, but not as a SAE in Denmark, as reported in the 2009 meta-analysis performed by Sin et al. ${ }^{13}$

\section{Results Studies}

Of the 43 potentially relevant studies identified in the database of AstraZeneca studies, 11 met the eligibility criteria for the pooled analysis. Studies were excluded due to lack of a relevant comparison $(n=11)$, crossover design $(n=7)$, duration $\leq 8$ weeks $(n=7)$, fewer than 60 total patients $(n=5)$, or open label design $(n=2)$. The studies in the analysis included patients treated with budesonide/formoterol, budesonide, formoterol, or placebo at a range of different doses (Table 1). ${ }^{14-24}$ The detailed methodology of these studies has been published elsewhere, with a brief summary provided in Table 1 . The daily delivered dose of inhaled budesonide in these studies ranged from 320 to $1,280 \mu \mathrm{g}$, with $640 \mu \mathrm{g}$ being the most common daily delivered dose. The duration of studies ranged from 3 to 12 months in studies of budesonide/formoterol, and 6-48 months in studies of budesonide.

\section{Patients}

The eleven studies included 10,574 randomized patients, of whom 10,570 received at least one dose of the study treatment and were included in the safety analysis set (budesonidecontaining treatments, $\mathrm{n}=5,750$; non-budesonide-containing treatments, $n=4,820$ ). The total exposure for the primary 


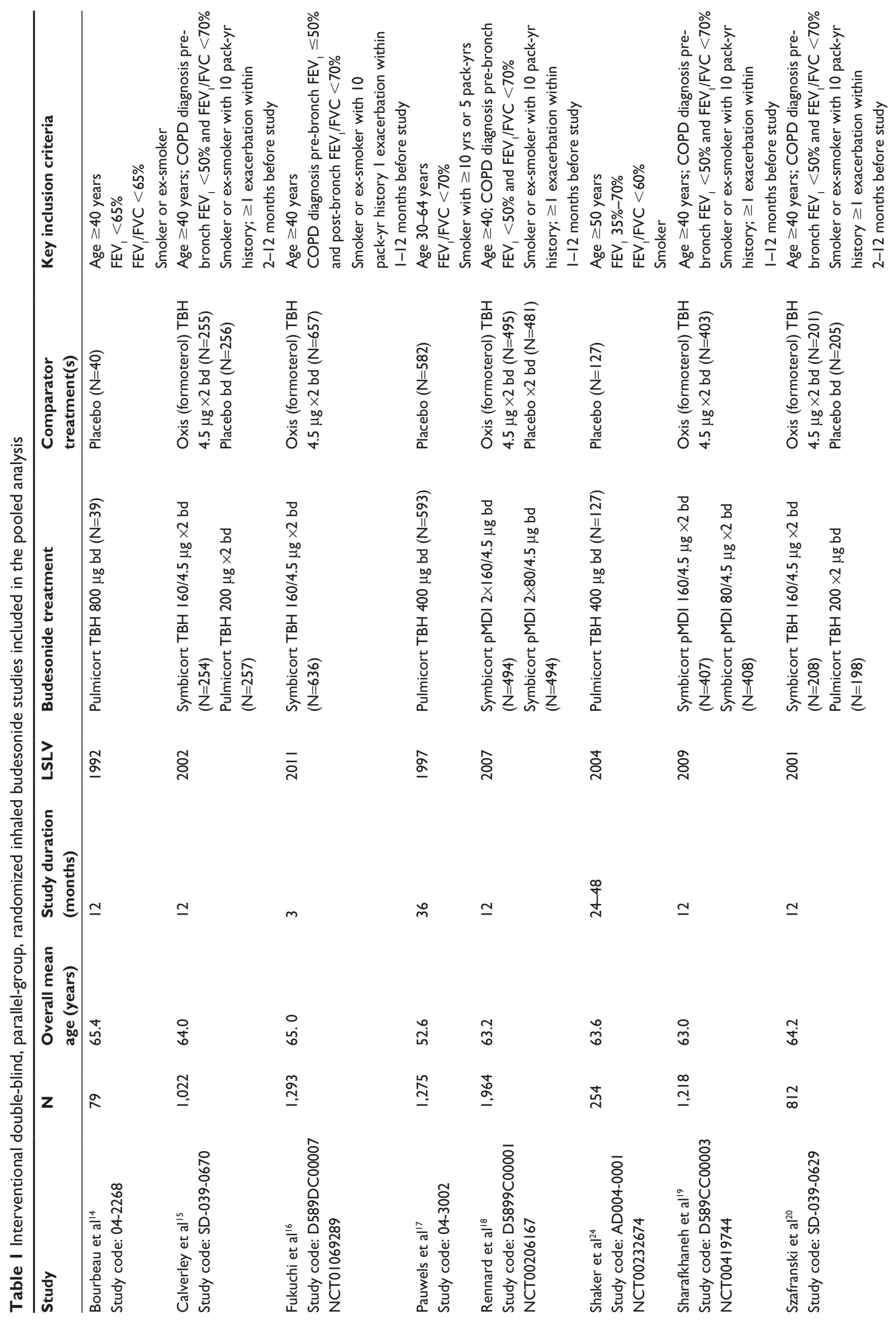



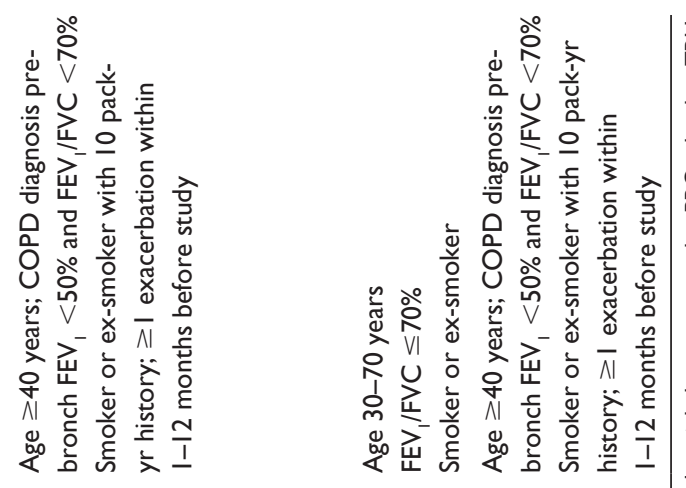

treatment comparison was 5,127 patient-years for the budesonide-containing treatments and 4,327 patient-years for the non-budesonide-containing treatments. There was around $10 \%$ additional average exposure per patient in the budesonide and budesonide/formoterol arms compared with the placebo arms (budesonide versus placebo, seven studies, median $9 \%$, range $1 \%-16 \%$; budesonide + formoterol versus placebo, five studies, median $12 \%$, range $0 \%-18 \%$ ).

The baseline patient characteristics of the included patients are shown in Table 2. In general, the age, smoking history, and lung function profiles of the patients included in the analysis were consistent with a population of patients with COPD In two of the early budesonide studies, ${ }^{17,22}$ more than half of patients had post-BD $\mathrm{FEV}_{1}>80 \% \mathrm{PN}$, indicating a milder patient population. In contrast, all of the other studies included less than $1 \%$ of patients with post-BD FEV ${ }_{1}>80 \% \mathrm{PN}$. These two studies also included a greater proportion of patients under the age of $55\left(55.3 \%{ }^{17}\right.$ and $\left.26.2 \%{ }^{22}\right)$, with the latter study including no patients over the age of 75 . In all of the other studies, $<20 \%$ of subjects were under the age of 55 . Two studies ${ }^{17,24}$ included only current smokers, and another study ${ }^{22}$ included predominantly current smokers (76.2\%); other studies included between $34 \%$ and $44 \%$ of current smokers. Thus, the designs of the included studies and the patient characteristics show some heterogeneity.

\section{Primary outcome variable: time to first pneumonia TESAE}

Overall, the results of this pooled analysis demonstrated no statistically significant increase in the risk of pneumonia TESAEs with the use of inhaled budesonide-containing treatments in patients with COPD, compared with non-budesonidecontaining treatments (Figure 1). The overall pooled hazard ratio (HR), comparing all patients receiving budesonidecontaining treatments (with or without formoterol) with all patients receiving non-budesonide-containing treatments (placebo or formoterol), was 1.15 (95\% CI: 0.83, 1.57) for pneumonia TESAEs. Descriptive statistics for pneumonia TESAEs and TEAEs in the pooled analysis by treatment are shown in Table 3 (primary treatment comparison) and Table S2 (secondary treatment comparisons). The incidence rates of pneumonia TESAEs with budesonide-containing treatments and non-budesonide-containing treatments were $1.9 \%$ and $1.5 \%$ per year, respectively.

The pooled HR for budesonide/formoterol-containing treatments versus non-budesonide-containing treatments was 1.00 (95\% CI: $0.69,1.44)$. The pooled HR for the comparison of budesonide alone with placebo was 1.57 


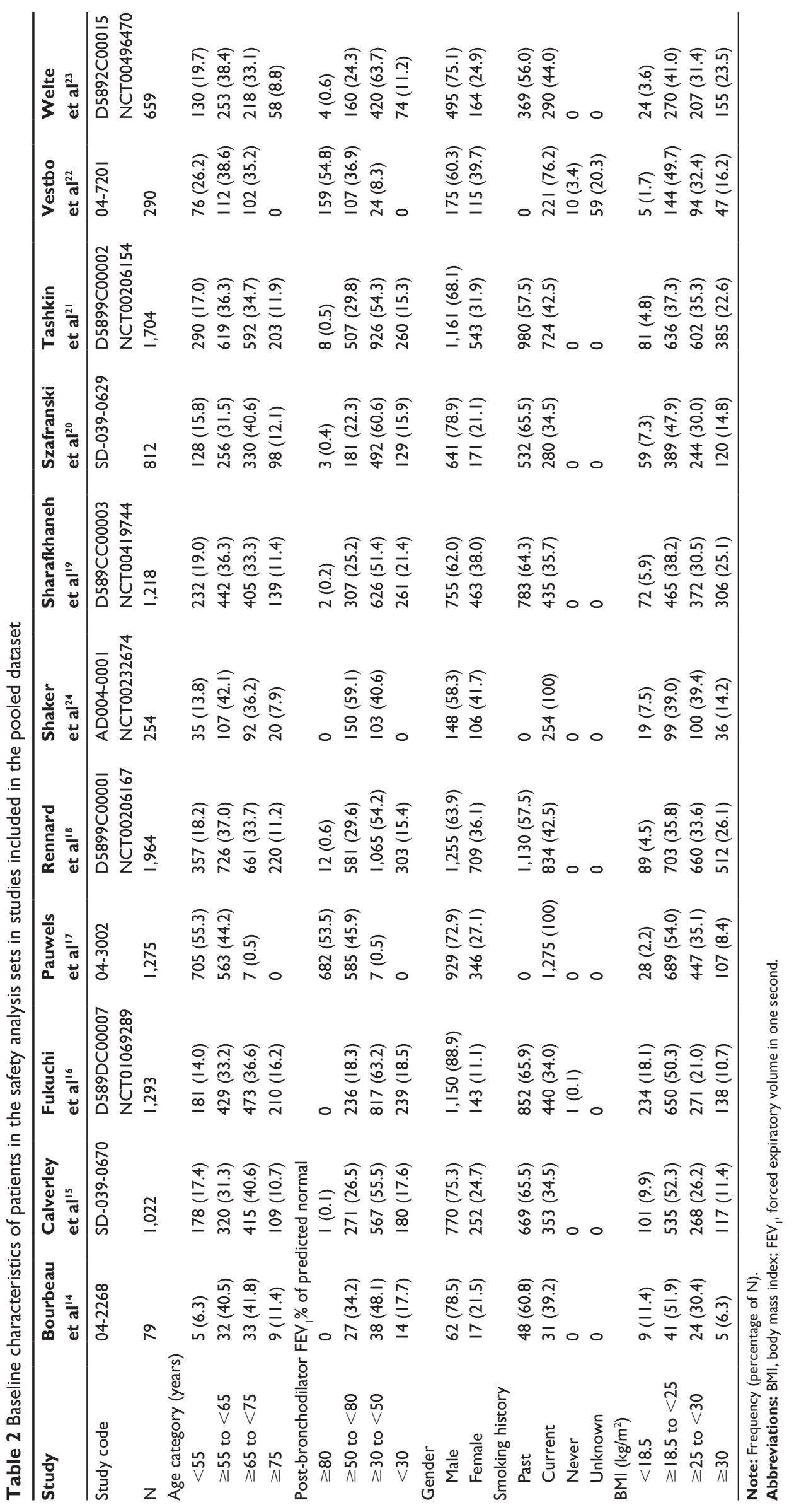




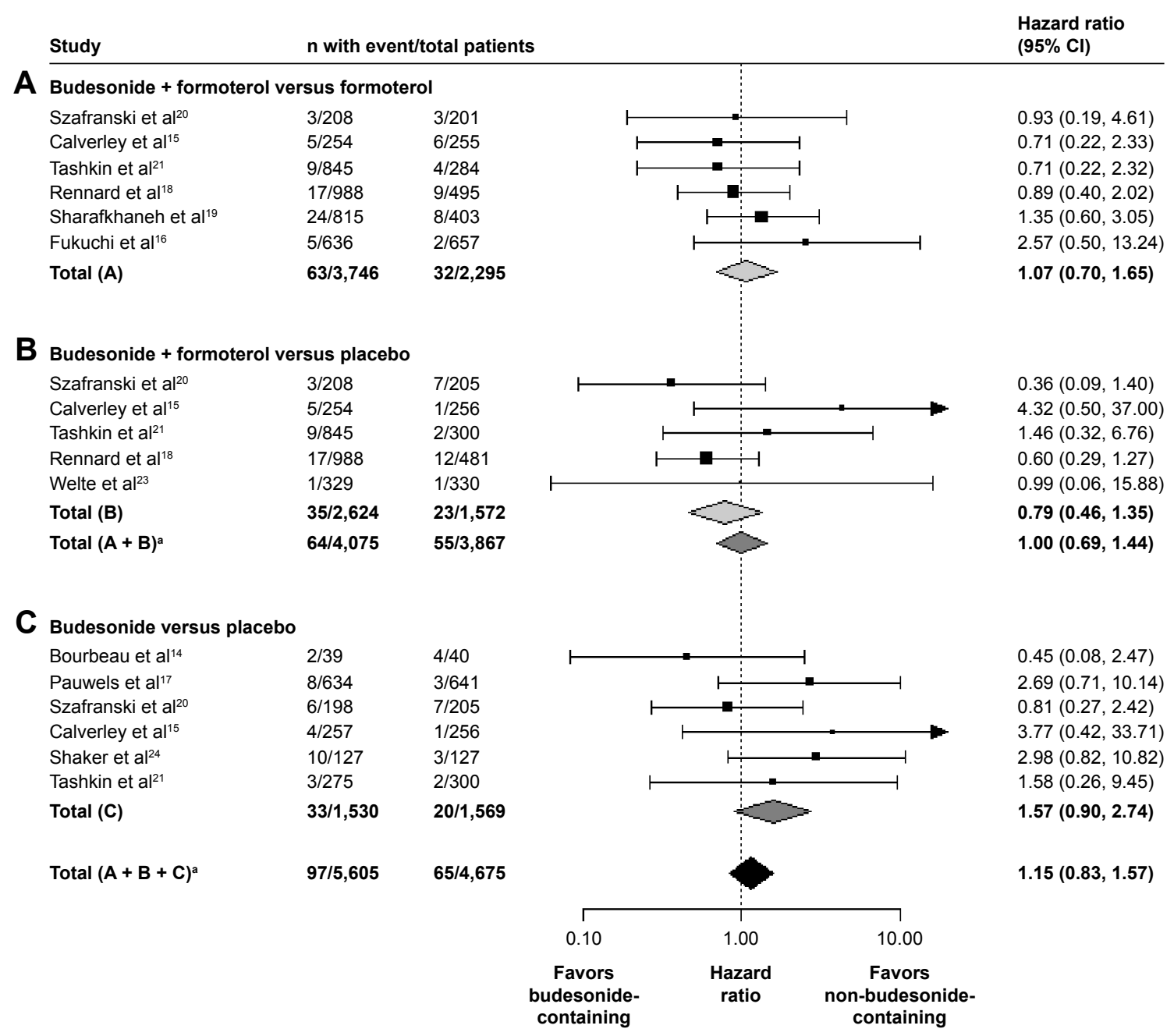

Figure I (A-C) Forest plot of hazard ratios for the effect of budesonide on pneumonia TESAEs.

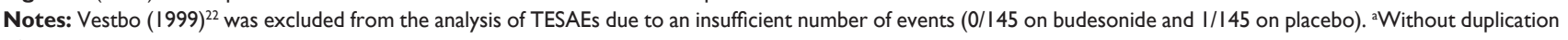
of shared arms.

Abbreviations: $\mathrm{Cl}$, confidence interval; TESAE, treatment-emergent serious adverse event.

Table 3 Pneumonia event comparisons in the pooled analysis

\begin{tabular}{|c|c|c|}
\hline & $\begin{array}{l}\text { Budesonide- } \\
\text { containing }\end{array}$ & $\begin{array}{l}\text { Non- } \\
\text { budesonide- } \\
\text { containing }\end{array}$ \\
\hline Safety analysis set $(\mathrm{N})$ & 5,750 & 4,820 \\
\hline Exposure (patient years) ${ }^{\mathrm{a}}$ & $5,127.2$ & $4,327.3$ \\
\hline Patients with pneumonia & $97(1.69)$ & $66(1.37)$ \\
\hline \multicolumn{3}{|l|}{ TESAEs, n (\%) } \\
\hline Rate/patient year & 0.019 & 0.015 \\
\hline Pneumonia TESAEs, $\mathrm{n}$ & 110 & 68 \\
\hline Rate/patient year & 0.021 & 0.016 \\
\hline Patients with pneumonia & $269(4.68)$ & 197 (4.09) \\
\hline \multicolumn{3}{|l|}{ TEAEs, n (\%) } \\
\hline Rate/patient year & 0.052 & 0.046 \\
\hline Pneumonia TEAEs, $n$ & 383 & 268 \\
\hline Rate/patient year & 0.075 & 0.062 \\
\hline Patients with fatal pneumonia, $n$ & 6 & 3 \\
\hline \multicolumn{3}{|c|}{$\begin{array}{l}\text { Notes: axposure is defined as time from date of first dose of randomized treatment } \\
\text { to date of last dose of randomized treatment. Incident rates for each treatment group } \\
\text { calculated as the number of patients experiencing an event divided by total exposure } \\
\text { in years. For event rates, the total number of events was used in the numerator. } \\
\text { Abbreviations: TESAE, treatment-emergent serious adverse event; TEAE, } \\
\text { treatment-emergent adverse event. }\end{array}$} \\
\hline
\end{tabular}

(95\% CI: 0.90, 2.74), but did not reach statistical significance despite being numerically larger than the overall estimate and the other subcomparison estimates. The incidence rate of pneumonia TESAEs was $1.2 \%$ per year on budesonide and $0.8 \%$ per year on placebo (Table S2). There was no statistical evidence of heterogeneity for TESAEs $(P>0.20)$; the associated $I^{2}$ statistics for all comparisons were $0 \%$, with high upper confidence limits ( $>71 \%)$.

A total of nine fatal pneumonia TESAEs were observed in the 10,570 patients included in the safety analysis; six in the budesonide-containing treatment arms and three in the non-budesonide-containing treatment arms.

\section{Secondary outcome variable: time to first pneumonia TEAE}

The pattern of results observed was generally similar to the primary endpoint. Overall, no statistical difference was observed in the risk for pneumonia TEAEs between patients 
receiving budesonide-containing and non-budesonidecontaining treatments (pooled HR: 1.13; 95\% CI: 0.94, 1.36; Figure 2). The incidence rates of pneumonia TEAEs with budesonide-containing treatments and non-budesonidecontaining treatments were similar $(5.2 \%$ and $4.6 \%$ per year, respectively).

The pooled HR for pneumonia TEAEs for the budesonide/ formoterol-containing treatments versus the non-budesonidecontaining treatments was 1.21 (95\% CI: 0.93, 1.57). As seen with the pneumonia TESAEs, the risk of experiencing a pneumonia TEAE was lower on budesonide/formoterol treatment versus placebo (HR: 0.93; 95\% CI: 0.64, 1.34), although the result was not statistically significant. A statistically significant increase was observed in the risk of pneumonia TEAEs on comparing budesonide/formoterol with formoterol (HR: 1.40; 95\% CI: 1.02, 1.93). The pooled HR for pneumonia TEAEs for budesonide alone versus placebo was not statistically significant (HR: 1.07; 95\% CI: 0.83, 1.38).
There was some evidence of statistical heterogeneity for budesonide-containing versus non-budesonide-containing treatments and budesonide versus placebo (Likelihood Ratio Tests, $P=0.08$ and 0.07 respectively), in which Pauwels et al's study (Study 04-3002) ${ }^{17}$ was particularly influential; but not for the other comparisons $(P>0.20)$. As for TESAEs, the associated $I^{2}$ statistics for all comparisons were $0 \%$, with high upper confidence limits $(>74 \%)$.

\section{Effect of dose, treatment duration, and treatment device on pneumonia TESAEs and TEAEs}

The most common daily delivered dose for budesonide/ formoterol was $640 / 18 \mu \mathrm{g}$; however, a lower daily delivered dose of $320 / 18 \mu \mathrm{g}$ budesonide/formoterol was also investigated in three studies. ${ }^{18,19,21}$ For budesonide, the most commonly daily delivered dose was $640 \mu \mathrm{g}$; higher daily delivered doses of $1,280 \mu \mathrm{g}$ for 12 months $^{14}$ and $960 \mu \mathrm{g}$ for 6 months followed by $1,280 \mu \mathrm{g}$ for 30 months $^{22}$ were also assessed.

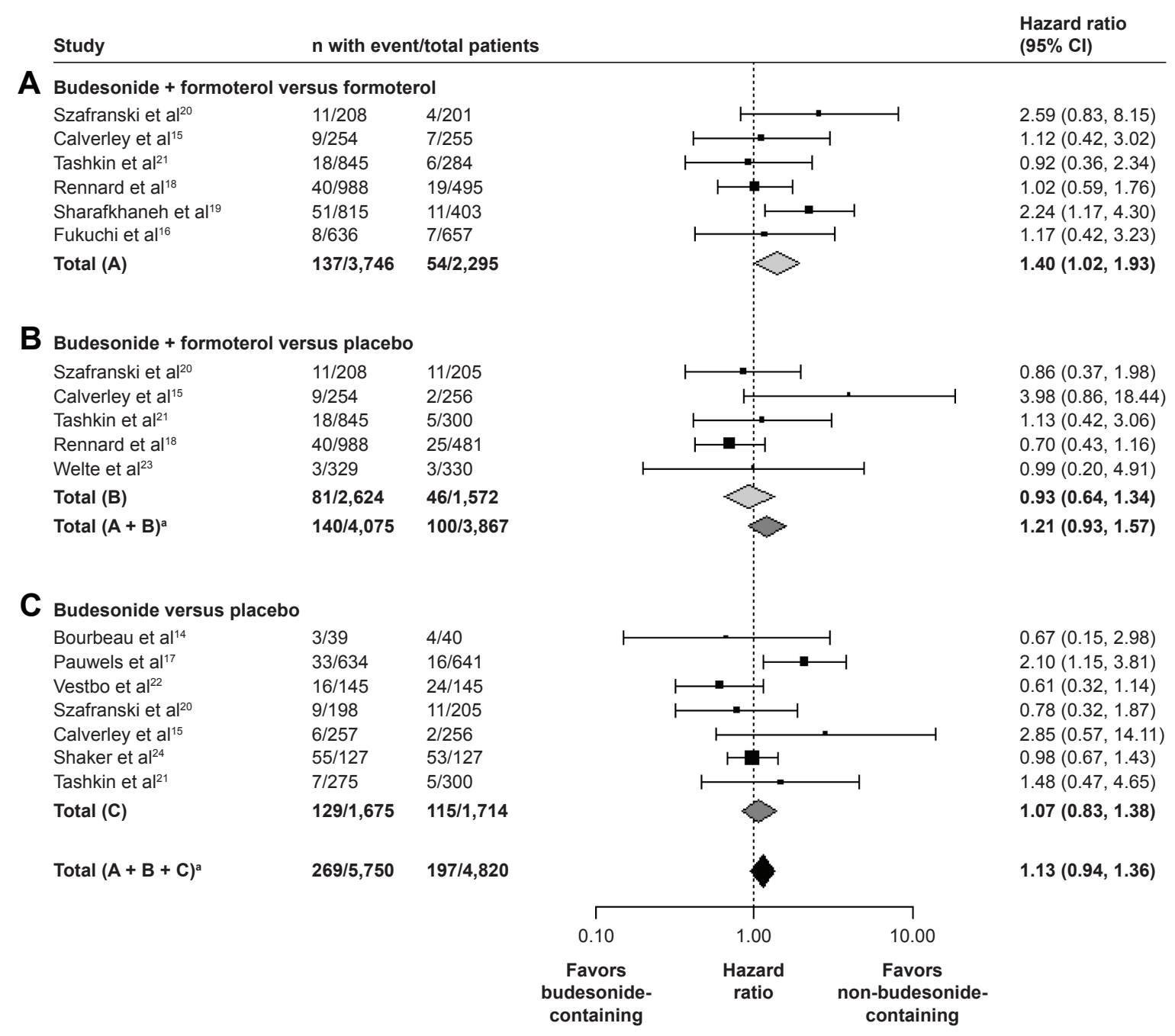

Figure 2 (A-C) Forest plot of hazard ratios for the effect of budesonide on pneumonia TEAEs.

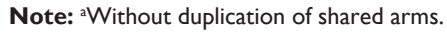

Abbreviations: $\mathrm{Cl}$, confidence interval; TEAE, treatment-emergent adverse event. 
In two pools of studies, a statistically significant increase was observed in the risk of pneumonia (budesonide $640 \mu \mathrm{g}$ versus placebo for TESAEs and budesonide/formoterol $640 / 18 \mu \mathrm{g}$ versus formoterol $18 \mu \mathrm{g}$ for TEAEs). However, there was no consistent dose-related effect across or within studies (Table S3).

Similarly for study duration, although the risk of pneumonia was observed to increase significantly for TESAEs for the pool of budesonide versus placebo greater than 12-month studies, and for TEAEs for the pool of budesonide/ formoterol versus formoterol 12-month studies, no consistent effect of treatment duration was observed across the studies (Table S4). There was no evidence of the treatment effect increasing or decreasing over time for the primary comparison of budesonide-containing versus non-budesonidecontaining treatments (time dependent covariate, $P=0.60$ ).
Using a piecewise Cox model, the point estimates for the HRs fluctuated over time (HRs of 0.94, 1.13, 0.54, and 2.13 for $0-<3,3-<6,6-12$, and $\geq 12$ months, respectively); there was no statistically significant evidence of increased risk of a TESAE for budesonide-containing versus non-budesonidecontaining treatment in any time interval.

There was no obvious effect of the device used for budesonide treatment on the risk of pneumonia TESAEs or TEAEs (Table S5).

\section{Effect of budesonide on the risk for pneumonia TESAEs and TEAEs in subgroups}

The HRs for pneumonia TESAEs (Figure 3) and TEAEs (Figure 4) with budesonide-containing versus non-budesonidecontaining treatments were estimated for subgroups using

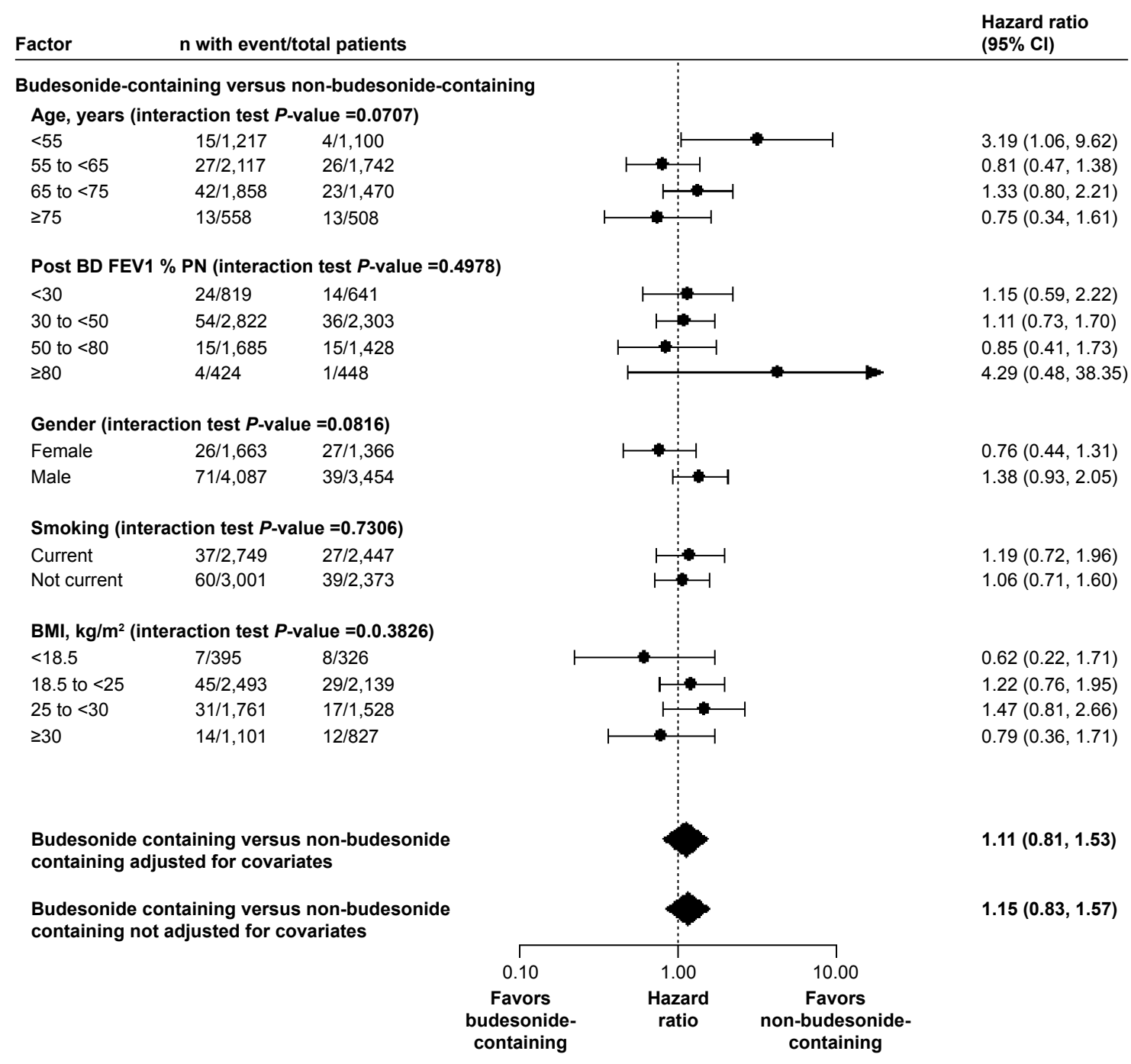

Figure 3 Forest plot of hazard ratios for the effect of budesonide on pneumonia TESAEs in risk factor subgroups.

Abbreviations: $\mathrm{BD}$, bronchodilator; $\mathrm{BMI}$, body mass index; $\mathrm{Cl}$, confidence interval; $\mathrm{FEV}_{1}$, forced expiratory volume in one second; PN, predicted normal; TESAE, treatmentemergent serious adverse event. 


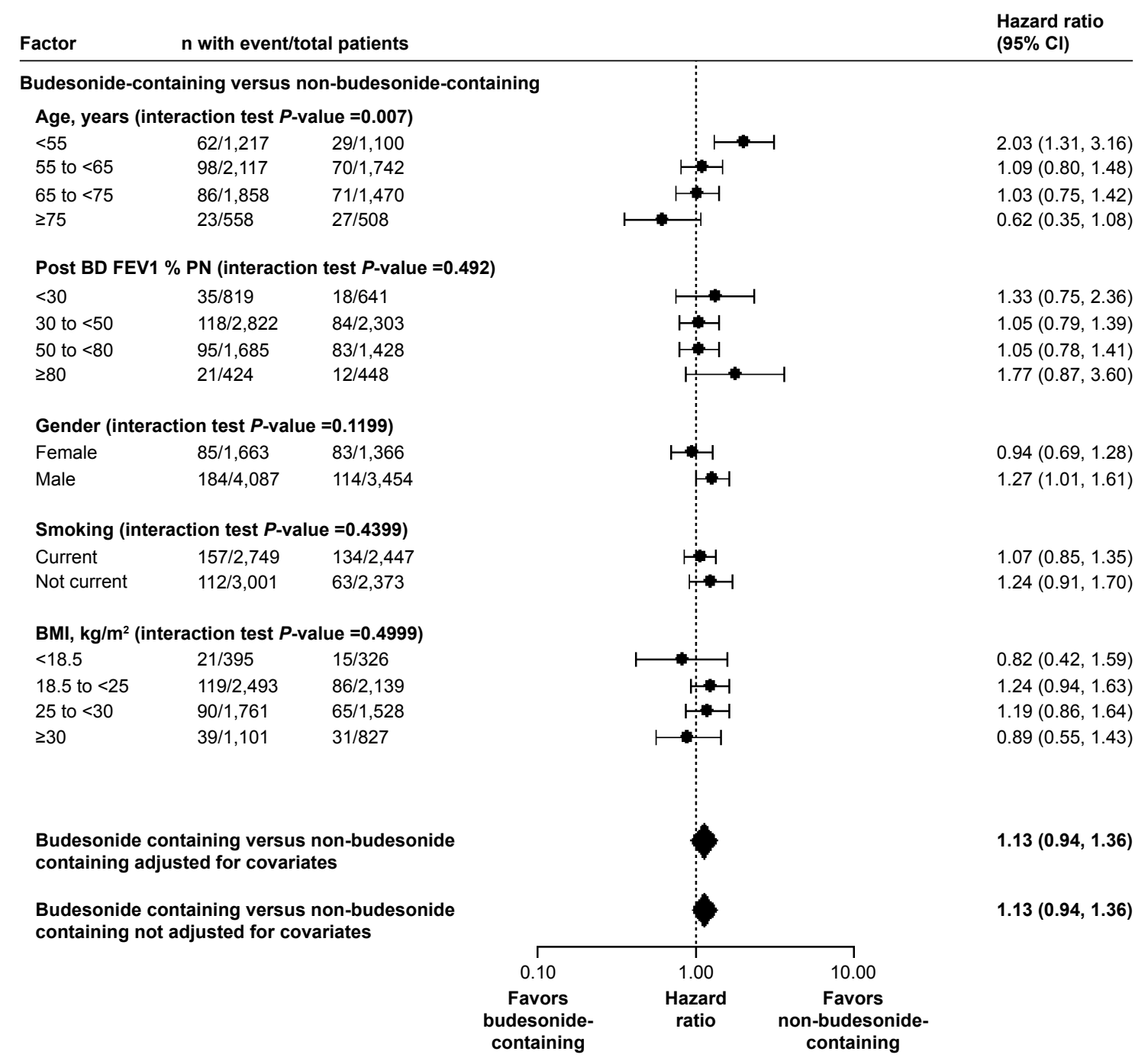

Figure 4 Forest plot of hazard ratios for the effect of budesonide on pneumonia TEAEs in risk factor subgroups.

Abbreviations: $\mathrm{BD}$, bronchodilator; $\mathrm{BMI}$, body mass index; $\mathrm{Cl}$, confidence interval; $\mathrm{FEV}_{\text {, }}$, forced expiratory volume in one second; PN, predicted normal; TEAE, treatmentemergent adverse event.

treatment-by-factor interactions for lung function (post-BD $\mathrm{FEV}_{1}$ at baseline), gender, age, smoking status, and BMI. There was statistical evidence of a differential treatment effect for budesonide-containing treatments versus nonbudesonide-containing treatments on the risk of pneumonia TESAEs and TEAEs across age groups (treatment-by-factor interaction: $P=0.07$ and 0.007 , respectively) and in TESAE risk by gender (treatment-by-factor interaction: $P=0.08$ ). A statistically significant increased risk of pneumonia TESAEs and TEAEs with budesonide was observed in patients aged $<55$ years. An increased risk of pneumonia TESAEs was seen in males with budesonide, whereas in females the trend was reversed, although the treatment effect was not statistically significant for either sex. A similar pattern of effects was observed for pneumonia TEAEs, although the interaction effect was not statistically significant (treatment-by-factor interaction: $P=0.12$ ). There was no evidence of a differential treatment effect on the risk of pneumonia across subgroups based on lung function, smoking status, or BMI at study entry.

\section{Effect of risk factors on the risk for pneumonia TESAEs and TEAEs}

The effects of risk factors on TESAE and TEAE risk are shown in Figures $\mathrm{S} 1$ and $\underline{\mathrm{S} 2}$ respectively. Increased age and lower post-BD FEV 1 were associated with increased TESAE risk, while current smokers displayed a lower TESAE risk compared with not-current smokers (HR: 0.63; 95\% CI: 0.44, 0.92). However, the effect of smoking was not statistically significant when the Cox model was extended to adjust for all factors simultaneously (data not shown). Increased age and lower post-BD FEV 1 were also associated with increased 
TEAE risk, and a trend for increased TEAE risk in females compared with males was observed (HR: 1.20; 95\% CI: 0.99, 1.45). The impact of gender persisted when the Cox model was extended to adjust for all factors (HR: 1.28; 95\% CI: $1.05,1.56)$. It should be noted that there were generally insufficient events within the risk factor categories within each study to usefully assess study-level risk factor effects.

\section{Post hoc sensitivity analysis}

In post hoc sensitivity analyses, excluding patients with post-BD $\mathrm{FEV}_{1} \geq 80 \% \mathrm{PN}$ or with post-BD $\mathrm{FEV}_{1} \geq 70 \%$ $\mathrm{PN}$, did not meaningfully affect the conclusions from the primary and secondary analyses. The removal of these patients resulted in a slightly reduced HR estimate for the primary treatment comparisons, but did not in general substantially affect the HR estimates. Of note, the HR for pneumonia TESAEs for budesonide versus placebo reduced from 1.57 (95\% CI: 0.90, 2.74) when patients with post-BD $\mathrm{FEV}_{1} \geq 70 \% \mathrm{PN}$ were included to 1.37 (95\% CI: 0.77, 2.46) when these patients were excluded.

In a separate post hoc sensitivity analysis for the comparison of budesonide-containing versus non-budesonidecontaining treatments, the HRs for pneumonia TESAEs remained very similar when patients from Danish centers were excluded, except for the budesonide versus placebo comparison where the HR decreased from 1.57 (95\% CI: $0.90,2.74)$ when patients from Danish centers were included to HR 1.14 (95\% CI: 0.60, 2.18) when they were excluded. For pneumonia TEAEs the HRs were also similar, except for the budesonide versus placebo comparison, where the HR increased from 1.07 (95\% CI: 0.83, 1.38) when patients from Danish centers were included to 1.50 (95\% CI: 0.93 , 2.45 ) when they were excluded. The incidence rate of pneumonia TEAEs was $4.8 \%$ per year on budesonide and $4.4 \%$ per year on placebo when patients from Danish centers were included, and $2.2 \%$ per year and $1.5 \%$ per year when they were excluded.

\section{Discussion}

This pooled individual patient-level analysis found no statistically significant increase in the overall risk of either pneumonia TESAEs or TEAEs in 10,570 patients with COPD receiving budesonide-containing compared with non-budesonide-containing treatments. The incidence rates of pneumonia AEs with budesonide- and non-budesonidecontaining treatments were similar $(1.9 \%$ and $1.5 \%$ per year, respectively, for pneumonia TESAEs, and 5.2\% and $4.6 \%$ per year, respectively, for pneumonia TEAEs).
Across the comparisons studied, the estimated HR for pneumonia TESAEs was numerically highest for budesonide versus placebo. This may be a random finding, due to the low number of patients experiencing an event, or may be driven by the greater heterogeneity in the patient populations in the early budesonide studies. Some of these early studies included only, or primarily, current smokers, and there was a general pattern of younger patients with milder COPD. In addition, all studies from the later budesonide/formoterol clinical development program required patients to have experienced a COPD exacerbation within the previous 12 months, whereas none of the studies from the earlier budesonide clinical development program had this criterion. Hence, the budesonide studies included patients who would not be treated with ICS according to current treatment guidelines. ${ }^{1}$ The incidence rate of pneumonia TESAEs was considerably lower in these patients, at $1.2 \%$ per year on budesonide and $0.8 \%$ per year on placebo.

When the current pooled data were analyzed with respect to dose and duration, while there were statistically significant increases in the risk of pneumonia events in some dose and duration categories, no consistent trend across dose or duration was observed across or within studies. However, there were no within-study comparisons of different dose levels, and only a narrow dose range of budesonide was available in these pooled studies; a limitation which applies to the majority of research assessing the effect of inhaled corticosteroid dose on pneumonia risk. In a nested case-control analysis of a new-user cohort of 163,514 COPD patients within Quebec health insurance databases, an increase in the risk of serious pneumonia (either requiring hospitalization or fatal) was dose-dependent with fluticasone but not with budesonide. ${ }^{6}$ Analyses of the effect of risk factors, dose, and treatment duration are based largely on crossstudy comparisons where these factors cannot be easily disentangled. Hence, no strong conclusions can be drawn on whether dose, treatment duration, or the risk factors analyzed have any impact on the risk of pneumonia, or the impact of budesonide on any risk of pneumonia. We are not aware of any precedent or obvious biological explanations for the observations of increased impact of budesonide on the risk of pneumonia in subgroups of male COPD patients or those under 55 years old. It is notable that there were only four pneumonia TESAEs in patients under 55 years old in the current non-budesonide-containing treatment pool.

As might be expected, the results of the current pooled analysis are similar to the previously published and subsequently updated pooled analysis of eight AstraZeneca trials 
by Sin et al. ${ }^{25}$ They compared 4,616 COPD patients who received inhaled budesonide (alone or in a fixed combination with formoterol) with 3,644 who received non-budesonide control treatment and found the occurrence of pneumonia, both as an $\mathrm{AE}$ and an SAE, to be similar across treatment groups. ${ }^{8,25}$ Key differences in the current pooled analysis are that pneumonia events were not censored at 12 months, a wider range of pneumonia preferred terms was included, and three additional studies were included. ${ }^{16,23,24}$

The most up to date and comprehensive published studylevel meta-analysis considering the risk of pneumonia with inhaled corticosteroids is the Cochrane review by Kew and Seniukovich. ${ }^{8}$ They examined the risk of pneumonia in patients with COPD treated with inhaled fluticasone or budesonide in randomized controlled studies of at least 12 weeks duration. Seventeen budesonide studies were identified, seven of which had data available for nonfatal pneumonia SAEs, for which a statistically significant increase was seen with inhaled budesonide versus controls (OR: 1.62; 95\% CI: 1.00-2.62). The current pooled analysis includes one study ${ }^{23}$ that is not included in Kew and Seniukovich, although it would appear to meet their inclusion criteria. Of the seven budesonide studies included in Kew and Seniukovich but not in the current pooled analysis, six of the studies ${ }^{26-31}$ included 50 patients or fewer and provided no pneumonia information for the analysis by Kew and Seniukovich. The remaining study was sponsored by Chiesi, ${ }^{32}$ and so individual patient level data were not available for our analyses. While the Kew and Seniukovich meta-analysis is rigorous in its statistical methodology, it is limited by the safety information available in study publications that provide the source data. Their analysis included 80 nonfatal pneumonia SAEs and one death due to pneumonia for inhaled budesonide versus controls, with the majority of the data including only the preferred term "pneumonia". In contrast, the pooled analysis presented here includes 163 pneumonia TESAEs, including a range of relevant preferred terms. Recent developments in clinical trial transparency should provide more consistent access to study and patientlevel AE data for future pooled analyses..$^{33}$

To our knowledge, this is the largest pooled analysis evaluating the risk of pneumonia at an individual level in patients with COPD receiving inhaled budesonide treatment. Studies from two separate clinical programs were included, spanning 22 years. Another key strength of this analysis is the primary focus on serious pneumonia AEs, which are medically more important, as well as having a less ambiguous diagnosis than nonserious pneumonia AEs. Furthermore, the use of individual patient-level data allows a consistent definition of pneumonia to be applied using a prespecified list of preferred terms. Finally, the use of a time-to-event analysis provides adjustment for the reduced exposure that is typically seen in the placebo arms of these studies due to differential withdrawal rates. However, there are some limitations with the analysis. These include the presence of substantial heterogeneity in study design in identifying pneumonia events and in patient characteristics within and across the 11 studies, and the low number of pneumonia TESAEs per study $(<40$; median: 12). The studies included in this analysis were not specifically designed to assess the risk of pneumonia events, which were identified based upon preferred terms from $\mathrm{AE}$ reporting rather than a uniform definition involving radiologic or bacteriologic confirmation. It should be noted that none of the trials included in this pooled analysis required a chest radiograph of patients who potentially had pneumonia. Intercountry and interregion differences may also be present. Previous studies ${ }^{13,34,35}$ have reported a higher incidence of pneumonia AEs in Denmark compared with other countries, with no difference in pneumonia SAEs, probably due to lack of specificity in reporting in the local language. Of the 11 studies included in the pooled analysis, two ${ }^{22,24}$ were conducted in Denmark, and Danish centers were included in three other studies. ${ }^{17,18,20}$ Reliability of the risk factor analyses could be another limitation. Several of the risk factors were very highly correlated; for example, high proportions of patients aged $<55$ years were current smokers, and not all risk factor categories were present in each study. There were too few events per stratum to undertake a meta-analysis of the covariate and interaction effects across studies; hence, it is possible that pooling studies could create spurious relationships between risk factors and outcomes. Finally, given the large number of comparisons made in this analysis, there is an increased risk of false-positive findings.

There is considerable debate around whether the risk of pneumonia varies between the different ICSs used for COPD treatment, with budesonide generally demonstrating reduced risk compared with fluticasone. ${ }^{6,36,37}$ While this is beyond the scope of this study, it has been hypothesized that this difference in risk may be attributed to the pharmacokinetic and pharmacodynamic properties (related to differences in lipophilicity/hydrophilicity) of the different ICSs ${ }^{38-40}$ It has been postulated that while the immunomodulatory effects of ICSs have been demonstrated to reduce exacerbations in patients with COPD,${ }^{41}$ they also increase the pneumonia risk, and the increased immunosuppressant activity of fluticasone may result in a greater risk..$^{42,43}$ 


\section{Conclusion}

The results of this pooled analysis found no statistically significant increase in overall risk in either pneumonia TESAEs or TEAEs with budesonide-containing versus non-budesonide-containing treatments. However, a small increase in risk with budesonide-containing treatment cannot be ruled out. While health care professionals and patients with COPD should be aware of the potential increased risk of pneumonia with ICS-containing therapy, this finding needs to be put in context of the full benefits and risks. Indeed European authorities have stated that overall the benefits of ICS medicines in treating COPD continue to outweigh their risks, and so there should be no change to the way in which these medicines are used. ${ }^{44}$

\section{Acknowledgments}

Medical writing assistance was provided by Parita Sheth and Katharine Williams of inScience Communications, Springer Healthcare, and was funded by AstraZeneca. This paper was presented at the ATS 2016 congress (May 13-18; San Francisco, CA, USA) as a poster presentation with interim findings. The poster's abstract was published in "Poster Abstracts" in Am J Respir Crit Care Med. 2016;193:A6819.

Sally Hollis and Gunnar Martensson are no longer affiliated to AstraZeneca.

\section{Disclosure}

Carin Jorup, Sally Hollis, Gunnar Martensson, Pontus Regnell, and Göran Eckerwall are all current or former employees of AstraZeneca. Dan Lythgoe is employed as a statistical consultant to AstraZeneca for this and other respiratory projects.

\section{References}

1. Global Initiative for Chronic Obstructive Lung Disease (GOLD). Global Strategy for Diagnosis, Management, and Prevention of COPD; 2016. Available from: http://goldcopd.org/global-strategy-diagnosis-management-prevention-copd-2016/. Accessed April 2, 2016.

2. Calverley P, Pauwels R, Vestbo J, et al. Combined salmeterol and fluticasone in the treatment of chronic obstructive pulmonary disease: a randomised controlled trial. Lancet. 2003;361(9356):449-456.

3. Calverley PM, Anderson JA, Celli B, et al. Salmeterol and fluticasone propionate and survival in chronic obstructive pulmonary disease. N Engl J Med. 2007;356(8):775-789.

4. Singh S, Loke YK. Risk of pneumonia associated with long-term use of inhaled corticosteroids in chronic obstructive pulmonary disease: a critical review and update. Curr Opin Pulm Med. 2010;16(2): $118-122$.

5. Yang IA, Clarke MS, Sim EH, Fong KM. Inhaled corticosteroids for stable chronic obstructive pulmonary disease. Cochrane Database Syst Rev. 2012(7):CD002991.

6. Suissa S, Patenaude V, Lapi F, Ernst P. Inhaled corticosteroids in COPD and the risk of serious pneumonia. Thorax. 2013;68(11): 1029-1036.
7. Suissa S, Coulombe J, Ernst P. Discontinuation of inhaled corticosteroids in COPD and the risk reduction of pneumonia. Chest. 2015;148(5): $1177-1183$

8. Kew KM, Seniukovich A. Inhaled steroids and risk of pneumonia for chronic obstructive pulmonary disease. Cochrane Database Syst Rev. 2014;3:CD010115.

9. European Medicines Agency. Inhaled corticosteroids containing medicinal products indicated in the treatment of chronic obstructive pulmonary disease. 2015; Available from: http://www.ema.europa.eu/ema/index. jsp?curl=pages/medicines/human/referrals/Inhaled_corticosteroids_for_ chronic_obstructive_pulmonary_disease/human_referral_prac_000050. jsp\&mid=WC0b01ac05805c516f. Accessed July 2, 2016.

10. ICH Harmonised Tripartite Guideline. Clinical Safety Data Management: Definitions and Standards for Expedited Reporting E2A (1994; updated 2005). Available from: http://www.gmp-compliance. org/guidemgr/files/E2A_GUIDELINE.PDF. Accessed April 2, 2016.

11. Smith CT, Williamson PR, Marson AG. Investigating heterogeneity in an individual patient data meta-analysis of time to event outcomes. Stat Med. 2005;24(9):1307-1319.

12. Higgins JP, Thompson SG. Quantifying heterogeneity in a metaanalysis. Stat Med. 2002;21(11):1539-1558.

13. Sin DD, Tashkin D, Zhang X, et al. Budesonide and the risk of pneumonia: a meta-analysis of individual patient data. Lancet. 2009; 374(9691):712-719.

14. Bourbeau J, Rouleau MY, Boucher S. Randomised controlled trial of inhaled corticosteroids in patients with chronic obstructive pulmonary disease. Thorax. 1998;53(6):477-482.

15. Calverley PM, Boonsawat W, Cseke Z, Zhong N, Peterson S, Olsson H. Maintenance therapy with budesonide and formoterol in chronic obstructive pulmonary disease. Eur Respir J. 2003;22(6):912-919.

16. Fukuchi Y, Samoro R, Fassakhov R, et al. Budesonide/formoterol via Turbuhaler(R) versus formoterol via Turbuhaler(R) in patients with moderate to severe chronic obstructive pulmonary disease: phase III multinational study results. Respirology. 2013;18(5):866-873.

17. Pauwels RA, Löfdahl CG, Laitinen LA, et al. Long-term treatment with inhaled budesonide in persons with mild chronic obstructive pulmonary disease who continue smoking. European Respiratory Society Study on Chronic Obstructive Pulmonary Disease. $N$ Engl J Med. 1999; 340(25):1948-1953

18. Rennard SI, Tashkin DP, McElhattan J, et al. Efficacy and tolerability of budesonide/formoterol in one hydrofluoroalkane pressurized metereddose inhaler in patients with chronic obstructive pulmonary disease: results from a 1-year randomized controlled clinical trial. Drugs. 2009; 69(5):549-565.

19. Sharafkhaneh A, Southard JG, Goldman M, Uryniak T, Martin UJ. Effect of budesonide/formoterol pMDI on COPD exacerbations: a double-blind, randomized study. Respir Med. 2012;106(2): 257-268.

20. Szafranski W, Cukier A, Ramirez A, et al. Efficacy and safety of budesonide/formoterol in the management of chronic obstructive pulmonary disease. Eur Respir J. 2003;21(1):74-81.

21. Tashkin DP, Rennard SI, Martin P, et al. Efficacy and safety of budesonide and formoterol in one pressurized metered-dose inhaler in patients with moderate to very severe chronic obstructive pulmonary disease: results of a 6-month randomized clinical trial. Drugs. 2008; 68(14):1975-2000.

22. Vestbo J, Sørensen T, Lange P, Brix A, Torre P, Viskum K. Long-term effect of inhaled budesonide in mild and moderate chronic obstructive pulmonary disease: a randomised controlled trial. Lancet. 1999; 353(9167):1819-1823.

23. Welte T, Miravitlles M, Hernandez P, et al. Efficacy and tolerability of budesonide/formoterol added to tiotropium in patients with chronic obstructive pulmonary disease. Am J Respir Crit Care Med. 2009;180(8): $741-750$.

24. Shaker SB, Dirksen A, Ulrik CS. The effect of inhaled corticosteroids on the development of emphysema in smokers assessed by annual computed tomography. COPD. 2009;6(2):104-111. 
25. Sin D, Tashkin D, Radner F, et al. An update on the risk of pneumonia related to budesonide use in COPD: Pooled analysis. Am J Respir Crit Care Med. 2012;185:A2945.

26. Laptseva IM, Laptseva EA, Borshchevsky VV, Gurevich G, Kalechits O. Inhaled budesonide in the management of chronic obstructive pulmonary disease. Eur Respir J. 2002;20(Suppl 38):244s.

27. Mirici A, Bektas Y, Ozbakis G, Erman Z. Effect of inhaled corticosteroids on respiratory function tests and airway inflammation in stable chronic obstructive pulmonary disease. Clin Drug Investig. 2001;21(12): 835-842.

28. Ozol D, Aysan T, Solak ZA, Mogulkoc N, Veral A, Sebik F. The effect of inhaled corticosteroids on bronchoalveolar lavage cells and IL-8 levels in stable COPD patients. Respir Med. 2005;99(12):1494-1500.

29. Renkema TE, Schouten JP, Koëter GH, Postma DS. Effects of long-term treatment with corticosteroids in COPD. Chest. 1996;109(5): 1156-1162.

30. Senderovitz T, Vestbo J, Frandsen J, et al. Steroid reversibility test followed by inhaled budesonide or placebo in outpatients with stable chronic obstructive pulmonary disease. The Danish Society of Respiratory Medicine. Respir Med. 1999;93(10):715-718.

31. Yildiz F, Basyigit I, Yildirim E, Boyaci H, Ilgazli A. Does addition of inhaled steroid to combined bronchodilator therapy affect health status in patients with COPD? Respirology. 2004;9(3):352-355.

32. Calverley PM, Kuna P, Monsó E, et al. Beclomethasone/formoterol in the management of COPD: a randomised controlled trial. Respir Med. 2010;104(12):1858-1868.

33. European Federation of Pharmaceutical Industries and Associations (efpia) and Pharmaceutical Research and Manufacturers of America (PhRMA) 2013. Principles for Responsible Clinical Trial Data Sharing. Available from: http://www.phrma.org/sites/default/files/ pdf/PhRMAPrinciplesForResponsibleClinicalTrialDataSharing.pdf. Accessed April 2, 2016.

34. Pauwels RA, Sears MR, Campbell M, et al. Formoterol as relief medication in asthma: a worldwide safety and effectiveness trial. Eur Respir J. 2003;22(5):787-794.

35. Sheffer AL, Silverman M, Woolcock AJ, Díaz PV, Lindberg B, Lindmark B. Long-term safety of once-daily budesonide in patients with early-onset mild persistent asthma: results of the Inhaled Steroid Treatment as Regular Therapy in Early Asthma (START) study. Ann Allergy Asthma Immunol. 2005;94(1):48-54.
36. Halpin DM, Gray J, Edwards SJ, Morais J, Singh D. Budesonide/formoterol vs. salmeterol/fluticasone in COPD: a systematic review and adjusted indirect comparison of pneumonia in randomised controlled trials. Int J Clin Pract. 2011;65(7):764-774.

37. Roberts M, Mapel D, Petersen H, Blanchette C, Ramachandran S. Comparative effectiveness of budesonide/formoterol and fluticasone/salmeterol for COPD management. J Med Econ. 2011;14(6):769-776.

38. Miller-Larsson A, Jansson P, Runström A, Brattsand R. Prolonged airway activity and improved selectivity of budesonide possibly due to esterification. Am J Respir Crit Care Med. 2000;162(4 Pt 1): 1455-1461.

39. Crim C, Calverley PM, Anderson JA, et al. Pneumonia risk in COPD patients receiving inhaled corticosteroids alone or in combination: TORCH study results. Eur Respir J. 2009;34(3):641-647.

40. Janson $\mathrm{C}$, Larsson $\mathrm{K}$, Lisspers $\mathrm{KH}$, et al. Pneumonia and pneumonia related mortality in patients with COPD treated with fixed combinations of inhaled corticosteroid and long acting beta2 agonist: observational matched cohort study (PATHOS). BMJ. 2013;346:f3306.

41. Jen R, Rennard SI, Sin DD. Effects of inhaled corticosteroids on airway inflammation in chronic obstructive pulmonary disease: a systematic review and meta-analysis. Int J Chron Obstruct Pulmon Dis. 2012;7:587-595.

42. Ek A, Larsson K, Siljerud S, Palmberg L, et al. Fluticasone and budesonide inhibit cytokine release in human lung epithelial cells and alveolar macrophages. Allergy. 1999;54(7):691-699.

43. Latorre M, Novelli F, Vagaggini B, et al. Differences in the efficacy and safety among inhaled corticosteroids (ICS)/long-acting beta2agonists (LABA) combinations in the treatment of chronic obstructive pulmonary disease (COPD): Role of ICS. Pulmo Pharmacol Ther. 2015; 30:44-50.

44. EMA.PRACReviews Known Risk of Pneumonia with Inhaled Corticosteroids for Chronic Obstructive Pulmonary Disease; 2016. Available from: http://www.ema.europa.eu/docs/en_GB/document_library/Referrals_ document/Inhaled_corticosteroids_Article_31/Recommendation_ provided_by_Pharmacovigilance_Risk_Assessment_Committee/ WC500203472.pdf. Accessed April, 2016.
International Journal of COPD

\section{Publish your work in this journal}

The International Journal of COPD is an international, peer-reviewed journal of therapeutics and pharmacology focusing on concise rapid reporting of clinical studies and reviews in COPD. Special focus is given to the pathophysiological processes underlying the disease, intervention programs, patient focused education, and self management protocols.

\section{Dovepress}

This journal is indexed on PubMed Central, MedLine and CAS. The manuscript management system is completely online and includes a very quick and fair peer-review system, which is all easy to use. Visit http://www.dovepress.com/testimonials.php to read real quotes from published authors. 